\title{
The Interaction of Sodium Dodecylsulfate with (Hydroxypropyl)Cellulose
}

\author{
Françoise M. WINNIK ${ }^{\dagger}$ and Mitchell A. WINNIK* \\ Xerox Research Center of Canada, 2660 Speakman Drive, Mississauga, \\ Ontario L5K 2L1, Canada \\ *Department of Chemistry and Erindale College, University of Toronto, \\ 80 St. George St., Toronto, Ontario M5S 1A1, Canada
}

(Received November 6, 1989)

\begin{abstract}
Hydroxypropyl)cellulose (HPC) in aqueous solution interacts with sodium dodecylsulfate (SDS) to give micelle-like SDS clusters bound to the polymer at concentrations well below the critical micelle concentration of SDS itself. We have used the steady-state fluorescence quenching method (fluorophore: pyrene, quencher: benzophenone) to determine mean aggregation numbers ( $\bar{N}$, molecules of SDS per cluster) for the system. These $\bar{N}$ values vary with both surfactant and HPC concentrations. The data are consistent with a constant number of cluster binding sites per polymer. These clusters increase in size as the ratio of SDS to HPC is increased. This behavior is very different from that described for the interaction of SDS with poly(ethylene oxide).
\end{abstract}

KEY WORDS Fluorescence Quenching / Neutral Water-Soluble Polymer / Polymer-Surfactant Interaction / Micelle / Mean Aggregation Number / Pyrene, Benzophenone / Critical Micelle Concentration /

Several years ago we reported that the interaction of surfactants with (hydroxypropyl)cellulose (HPC), a hydrophobic watersoluble polymer, was different from their interaction with hydrophilic water-soluble polymers, such as poly(ethylene oxide) (PEO) and poly(vinyl pyrrolidone) (PVP). ${ }^{1}$ We used a fluorescent probe technique as well as fluorescence labeling experiments to show that sodium dodecylsulfate (SDS) forms mixed aggregates with HPC at lower concentration than with PEO or PVP. In addition we showed that cationic surfactants, such as cetyltrimethylammonium chloride, which do not interact with PEO or PVP ${ }^{2}$ also form mixed aggregates with HPC. More recently, Lindman and coworkers used pulsed gradient NMR measurements to establish that another hydrophobically modified cellulosic polymer, ethyl- (hydroxyethyl)cellulose, also interacts with a cationic detergent, dodecyltrimethylammonium bromide. ${ }^{3}$

One of the most fascinating discoveries about the interaction of PEO with SDS is the type of aggregation which occurs, and how the aggregate structures vary as the surfactant-topolymer ratio is changed. Elucidated largely by Cabane et $a l^{4}$ using NMR and neutron scattering techniques, the picture which emerges is one in which the surfactant forms stoichiometric complexes in the form of small spheres, $20 \AA$ in radius, adsorbed onto the PEO strands. The polymer becomes incorporated into the head group region of the SDS clusters. While these clusters are similar in size to pure SDS micelles, they have a lower critical aggregation concentration (cac), and a mean aggregation number $(\bar{N}=38 \pm 30 \%)$ signifi-

† To whom correspondence should be addressed. 
cantly smaller than that of pure SDS micelles ( $\bar{N}=60$ to 65 ). At low SDS concentrations, the polymer and surfactant are molecularly dispersed. Above the cac, stoichiometric complexes form. Increasing the SDS concentration leads to the formation of more clusters of similar size. The spacing of the micelles, at low ionic strength, is determined by an interplay between their mutual coulombic repulsion and their need to adhere to the polymer chain. Eventually the chains become saturated, at $90 \AA$ ( $c a .140$ monomer units) between adjacent micelles. When these concentrations exceed the normal critical micelle concentration $(\mathrm{cmc})$ of SDS, the solution contains SDS micelles in addition to the bound clusters.

The SDS/PEO system has also been studied by Zana $^{5}$ and by Witte and Engberts, ${ }^{6}$ using fluorescence quenching techniques to determine the mean aggregation number for the micelles as a function of SDS concentration. These experiments confirm the idea that SDS clusters bound to the PEO have lower $\bar{N}$ values than the pure SDS micelles themselves. Zana et al. observed for a PEO concentration of $1 \%$ by weight, that $\bar{N}$ increased from 25 to 60 , as the SDS concentration increased from $0.01 \mathrm{M}$ to $0.1 \mathrm{M}$. Since all of these concentrations are above the cmc of $\operatorname{SDS}\left(8.2 \times 10^{-3} \mathrm{M}\right){ }^{7}$ this increase in $\bar{N}$ is consistent with an experiment that samples both bound and free micelles, and becomes more heavily weighted toward the latter, as the SDS concentration is increased.

Here we also use fluorescence quenching techniques to determine the mean aggregation number for SDS clusters bound to HPC. We find a very different type of behavior. $\bar{N}$ values depend upon the SDS concentration, even well below the cmc of SDS where no pure SDS micelles are formed. The picture that emerges here is that above the cac, small mixed micelles form. As the SDS concentration increases, the average number of clusters remains constant but their mean size increases.

\section{EXPERIMENTAL}

\section{Materials}

Chemicals were purchased from Aldrich Chemical Co., except sodium dodecylsulfate (SDS purum), which was obtained from Fluka. (Hydroxypropyl) cellulose (HPC, Klucel L, Hercules) had a nominal molecular weight of 100,000. Pyrene (99\%) was purified by repeated recrystallizations from absolute ethanol and subsequent sublimation. Water was deionized with a Millipore Milli-Q water purification system.

\section{Instrumentation}

UV absorption spectra were recorded with a Hewlett-Packard 8450A diode array spectrometer. Steady-state fluorescence spectra were run on a SPEX Fluorolog 212 spectrometer equipped with a DM3000F data system. The temperature of the water-jacketted cell holder was controlled via a water-circulating bath. Sonications were performed in an Ultrasonic Cleaner Model 8845-44 from Cole-Parmer.

\section{Fluorescence Measurements}

Emission spectra, recorded at $25^{\circ} \mathrm{C}$, were obtained with the excitation wavelength set at $330 \mathrm{~nm}$ and slits of $3.6 \mathrm{~nm}$ (excitation) and $0.9 \mathrm{~nm}$ (emission). Samples were not degassed. The ratios of fluorescence intensities in the absence and presence of quencher (benzophenone) were calculated as the ratios of the integrated spectral areas (in wavelength units, from 360 to $460 \mathrm{~nm}$ ).

\section{Samples for Spectroscopic Analysis}

Solutions of HPC containing pyrene were prepared at room temperature by dissolving HPC in pyrene-saturated water, previously filtered to remove pyrene microcrystals. For measurements of $I_{1} / I_{3}$ as a function of surfactant concentration, solutions were prepared by adding sufficiently small aliquots of SDS solutions to the HPC-pyrene solutions, 
such that these were diluted by no more than $10 \%$. Solutions were prepared $2 \mathrm{~h}$ before spectroscopic analysis. Solutions for quenching experiments were prepared as follows. SDS was added to solutions of HPC $\left(50 \mathrm{ml}, 1.0 \mathrm{~g} \mathrm{l}^{-1}\right.$, or $0.3 \mathrm{gl}^{-1}$ ), so that the SDS concentration ranged from $1 \times 10^{-3} \mathrm{M}$ to $13 \times 10^{-3} \mathrm{M}$. A portion of these solutions was saturated with benzophenone by sonication $(30 \mathrm{~min})$ with solid benzophenone and subsequent filtration. Aliquots of the benzophenone-saturated solutions were added to solutions of HPC and SDS to cover a benzophenone concentration range of 0 to $20 \times 10^{-5} \mathrm{M}$ for each HPC/SDS solution. The concentration of benzophenone in each sample was calculated from its UV absorption at $255 \mathrm{~nm}\left(\varepsilon_{255 \mathrm{~nm}}=18,600\right),{ }^{8}$ dilutions being performed for solutions of absorbance at $255 \mathrm{~nm}$ greater than 1.2.

\section{RESULTS AND DISCUSSION}

\section{Critical Aggregation Concentration}

When SDS is added to a solution of HPC plus pyrene $\left(\mathrm{ca} .3 \times 10^{-7} \mathrm{moll}^{-1}\right)$ in water the fluorescence spectrum of pyrene changes once a critical surfactant concentration is exceeded. The spectrum shows a decrease of the $(0,0)$ band at $376 \mathrm{~nm}$ (intensity $I_{1}$ ) relative to that of the $(0,2)$ band at $386 \mathrm{~nm}\left(I_{3}\right)$. A decrease in $I_{1} / I_{3}$ reflects transfer of pyrene from a more polar to a less polar environment. It is well documented that this type of change commonly accompanies micelle formation, both in the absence and in the presence of polymer. ${ }^{9}$ Plots of $I_{1} / I_{3}$ for pyrene in aqueous HPC as a function of SDS concentration are shown in Figure 1. Interaction of the probe with SDS in the presence of HPC is first observed at a value of [SDS] of $c a .1 .3 \mathrm{mM}$. The inflection point of the curve corresponds to $[\mathrm{SDS}]=1.73 \mathrm{mM}$. This value is about 5 times smaller than the cmc of SDS itself. Saturation is achieved at $[\mathrm{SDS}]=6.3 \mathrm{mM}$. The measurements were performed at two different concentrations of HPC, $0.3 \mathrm{gl}^{-1}$ and $1.0 \mathrm{gl}^{-1}$. In this range, the value

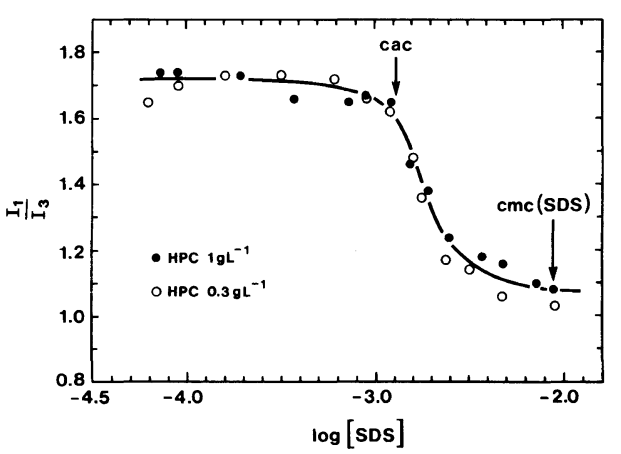

Figure 1. $I_{1} / I_{3}$ intensity ratio for pyrene $\left(\mathrm{ca} .3 \times 10^{-7} \mathrm{M}\right)$ fluorescence in aqueous solutions of HPC as a function of SDS concentration: HPC concentration, $1 \mathrm{gl}^{-1}$ (full circles); $0.3 \mathrm{~g}^{-1}$, (open circles).

of the cac does not seem to be affected by polymer concentration.

\section{Mean Aggregation Numbers}

Mean aggregation numbers $\bar{N}$ were determined by the steady state fluorescence quenching method with pyrene as the fluorophore $(F)$ and benzophenone as the quencher $(Q)$. This method works well when the following five criteria are met: 1) the micelles are not too large $(\bar{N}<100) ; 2)$ the fraction of both $F$ and $Q$ located in the aqueous phase is negligibly small; 3) $F$ and $Q$ do not form ground-state complexes; 4) $Q$ is a very efficient quencher of $F^{*}$ (i.e., diffusion-controlled quenching); and 5) at the concentrations employed, $Q$ has a negligible absorbance at the wavelength used to excite $F{ }^{6}$ Under these circumstances, the fluorescence intensity $I$ depends upon the concentration [Q] according to the expression:

$$
\ln \frac{I^{0}}{I}=\frac{[\mathrm{Q}]}{[\text { clusters }]}=\frac{\bar{N}[\mathrm{Q}]}{[\mathrm{SDS}]-[\mathrm{CAC}]}
$$

Here the intensity $I^{0}$ is the fluorescence intensity in the absence of quencher, [SDS] is the total concentration of SDS, and [CAC] is taken to be the concentration of free surfactant molecules not incorporated into clusters or micelles. 
The benzophenone-pyrene pair satisfy these experimental criteria. ${ }^{13}$ In our hands this $F / Q$ pair yielded the correct aggregation number for pure SDS micelles, $\bar{N}=59$, (reported value, $c a$. $60)^{14}$ and a reasonable value for micelles of cetyltrimethylammonium chloride, $\bar{N}=69$, (reported value, $c a .80) .{ }^{15}$ Note that the values of $\bar{N}$ calculated from the slopes of these lines depend sensitively on the value chosen for [CAC], when the total SDS concentration is close to the cac, but this problem becomes less important as the value of [SDS] becomes larger.

When the method was applied to the SDS-HPC system, the fluorescence quenching results showed a linear regime with a slight downward curvature at elevated quencher concentrations. The linear part corresponds to the region where the Poisson statistics assumed in the derivation of eq 1 are valid (Figure 2). The lines drawn through the linear portion of the data points correspond to the best fit. From these data we obtain two surprising result. The first is that $\bar{N}$ increases with increasing [SDS] (Figure 3) and passes through a maximum at an SDS concentration just above the $\mathrm{cmc}$ of SDS itself. The second surprise is that $\bar{N}$ depends also on the HPC concentration, or more precisely on the ratio of SDS to HPC. This result is apparent in Figure 3, but is also emphasized in Figure 2, where we show that quenching experiments which employ identical SDS concentrations, but different HPC concentrations, give different slopes when the data are plotted according to eq 1 . These two observations are in striking contrast to Cabane's findings for the SDSPEO system. In that system, the number of SDS clusters and the size of these clusters were unaffected by added PEO. It is clear from these two sets of data that the interaction of SDS with HPC is fundamentally different from its interaction with PEO.

\section{Model for the HPC-SDS Interaction}

(Hydroxypropyl)cellulose differs from poly(ethylene oxide) in three important ways. First,

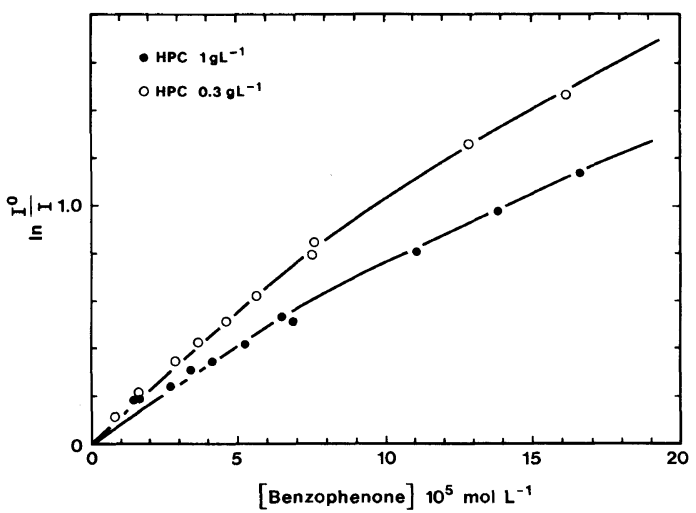

Figure 2. Plots of $\ln \left(I^{0} / I\right)$ for pyrene $\left(c a .3 \times 10^{-7} \mathrm{M}\right)$ in aqueous solutions of HPC and SDS as a function of benzophenone concentration: $\mathrm{HPC}=0.3 \mathrm{gl}^{-1}$; $[\mathrm{SDS}]=$ $6.8 \times 10^{-3} \mathrm{moll}^{-1}$ (open circles); $\mathrm{HPC}=1.0 \mathrm{gl}^{-1}$; $[\mathrm{SDS}]=$ $6.8 \times 10^{-3} \mathrm{moll}^{-1}$ (full circles).

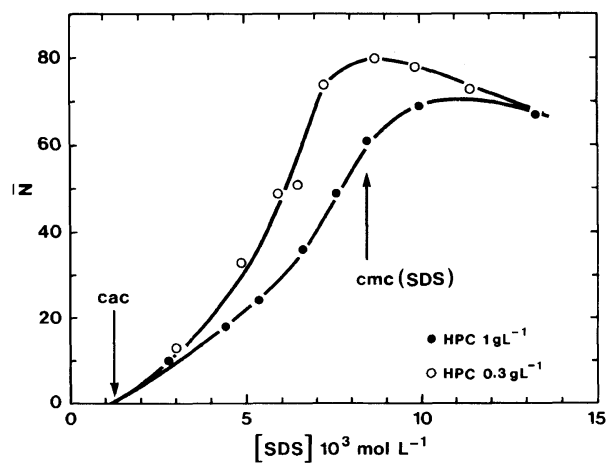

Figure 3. Plot of the aggregation number $\bar{N}$ of SDS-HPC clusters as a function of SDS concentration: HPC concentration, $1.0 \mathrm{gl}^{-1}$ (Full circles); $0.3 \mathrm{gl}^{-1}$ (open circles).

HPC is a hydrophobic polymer. Estimates of its Hildebrand solubility parameter $\left(\delta_{\mathrm{H}}\right)$ yield values on the order of $11\left(\mathrm{cal} / \mathrm{cm}^{3}\right)^{1 / 2},{ }^{16}$ while water has a value of $23\left(\mathrm{cal} / \mathrm{cm}^{3}\right)^{1 / 2}$. Polymers normally do not dissolve in solvents which differ by more than 2 to $3 \delta_{\mathrm{H}}$ units. HPC is solubilized in water because of the cooperative nature of its hydrogen bonding to water. Like PEO solutions, HPC aqueous solutions have a lower critical solution temperature (LCST) at which they undergo phase separation, but the LCST for HPC, at $42^{\circ} \mathrm{C}$, lies more than $50^{\circ} \mathrm{C}$ below that of PEO. 


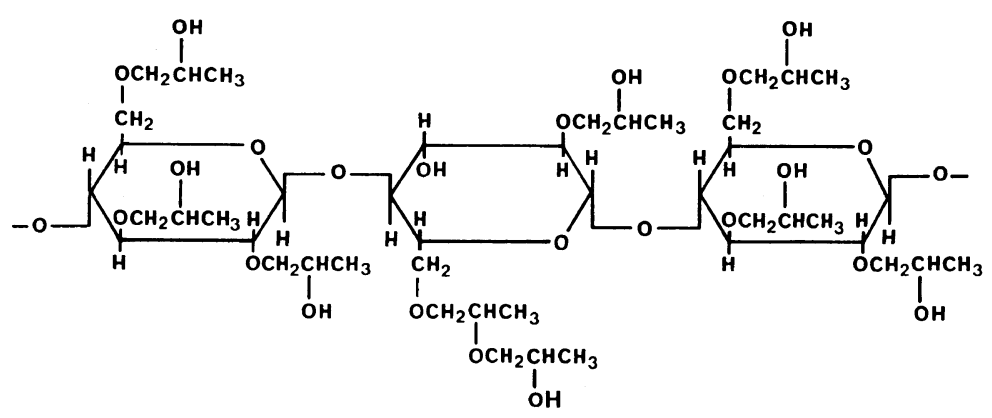

Figure 4. Idealized structure of (hydroxypropyl)cellulose with a degree of substitution of 3.3. Note both the free glucose hydroxyl group and the oligopropylene oxide chain attached to the polymer.

A second difference is that of chain stiffness. Cellulose derivatives are locally stiff, with rather large persistence lengths. ${ }^{17}$ The monomer unit of HPC is much larger than that of PEO. These factors must lead to differences in the details of binding of the two polymers with clusters of SDS. The third difference is that HPC is in fact a rather heterogeneous material. Hydroxypropyl groups are attached to most (but not all) of the glucose hydroxyls. In addition some short oligomeric sequences of poly(propylene oxide) protrude from the cellulose backbone (see Figure 4). We know that the overall degree of hydroxypropylation of the HPC employed is 3.3 per glucose unit, but we have no information about the distribution of these substituents. This heterogeneity opens the possibility that there are preferred binding sites on the polymer for surfactants.

The mean numbers of SDS clusters per polymer can be calculated from $\bar{N}$. These are reported in Table $I$ in terms of the number of glucose monomeric units per cluster, assuming $M_{n}=36,000,{ }^{18}$ e.g., on average 107 glucose units per polymer chain. We find that the mean number of clusters per chain seems to remain constant with increasing surfactant concentration. The clusters grow in size as more SDS is added to the system and decrease in size when HPC is added.

At SDS concentrations above the $\mathrm{cmc}$ of pure SDS, the bound clusters are larger in size
Table I. Mean Aggregation Numbers and Mean Number of Surfactant Clusters in the SDS-HPC system

a) $\mathrm{HPC}=1.0 \mathrm{gl}^{-1}$

\begin{tabular}{|c|c|c|c|}
\hline [SDS] & \multirow{2}{*}{$\bar{N}$} & [SDS Clusters] & \multirow{2}{*}{$\begin{array}{l}\text { Number of glucose } \\
\text { units per SDS clusters }\end{array}$} \\
\hline $\mathrm{moll}^{-1}$ & & $\mathrm{moll}^{-1}$ & \\
\hline $2.82 \times 10^{-3}$ & 10 & $1.5 \times 10^{-4}$ & 19 \\
\hline $4.43 \times 10^{-3}$ & 18 & $1.7 \times 10^{-4}$ & 17 \\
\hline $5.37 \times 10^{-3}$ & 24 & $1.7 \times 10^{-4}$ & 17 \\
\hline $6.64 \times 10^{-3}$ & 38 & $1.5 \times 10^{-4}$ & 19 \\
\hline $7.60 \times 10^{-3}$ & 49 & $1.3 \times 10^{-4}$ & 23 \\
\hline $8.47 \times 10^{-3}$ & 61 & $1.3 \times 10^{-4}$ & 23 \\
\hline
\end{tabular}

b) $\mathrm{HPC}=0.3 \mathrm{gl}^{-1}$

\begin{tabular}{|c|c|c|c|}
\hline [SDS] & \multirow{2}{*}{$\bar{N}$} & [SDS Clusters] & \multirow{2}{*}{$\begin{array}{l}\text { Number of glucose } \\
\text { units per SDS clusters }\end{array}$} \\
\hline $\mathrm{moll}^{-1}$ & & $\mathrm{moll}^{-1}$ & \\
\hline $3.0 \times 10^{-3}$ & 13 & $1.3 \times 10^{-4}$ & 22 \\
\hline $4.86 \times 10^{-3}$ & 33 & $1.1 \times 10^{-4}$ & 36 \\
\hline $5.90 \times 10^{-3}$ & 49 & $0.9 \times 10^{-4}$ & 32 \\
\hline $6.48 \times 10^{-3}$ & 51 & $1.1 \times 10^{-4}$ & 26 \\
\hline $7.25 \times 10^{-3}$ & 74 & $0.8 \times 10^{-4}$ & 36 \\
\hline $8.71 \times 10^{-3}$ & 80 & $0.9 \times 10^{-4}$ & 32 \\
\hline
\end{tabular}

than SDS micelles, and there is a competition between polymer-bound clusters and SDS micelle formation. As the SDS concentration is increased even further, large numbers of pure SDS micelles form. The fluorescence quenching experiment samples both the bound clusters and the free micelles, so that $\bar{N}$ becomes heavily 
(a)

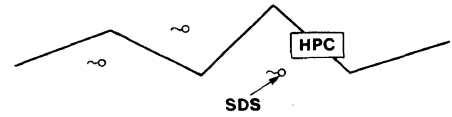

(b)

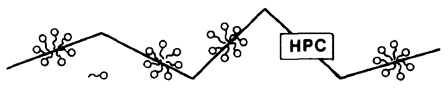

(c)

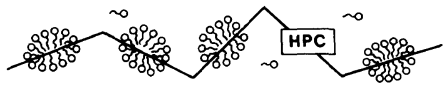

(d)

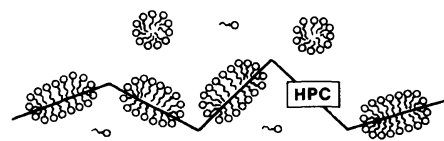

Figure 5. Model for the interactions between HPC and SDS. The HPC is represented as five Kuhn lengths. In (a), [SDS] < cac, HPC and molecularly dispersed SDS are present. In (b) and (c), cac $<$ [SDS $]<\mathrm{cmc}_{(\mathrm{SDS})}$, polymerbound surfactant clusters form and grow in size. In (d), [SDS] $>\mathrm{cmc}_{(\mathrm{SDS})}$, cluster size continues to increase; free [SDS] micelles are also formed.

weighted toward the contribution of the latter. This description explains the maxima shown in Figure 3 and the tendency for $\bar{N}$ to approach 60 at high concentrations of SDS.

In summary, our experiments suggest the following picture of the SDS-HPC interactions (Figure 5). At low concentrations, both species are molecularly dispersed. Above the cac small clusters of SDS form on the polymer. These grow in size as more SDS is added, and, it appears, no new SDS clusters add to the polymer chain. Above the $\mathrm{cmc}$ of SDS, the clusters continue to grow in size, but they are now in competition with the formation of SDS micelles. This situation is very different from that of the SDS-PEO interaction. Here, above the cac, spherical SDS aggregates of unique stoichiometry bind to the polymer. Introduction of additional SDS into the system leads to the formation of a larger number of similar-size polymer-bound clusters which become more closely spaced until the polymer chain is saturated. In both systems, the forces operating are identical, hydrophobic forces promoting association and clustering of the hydrocarbon portion of the surfactant, electrostatic repulsion between the polymer-bound clusters, and weaker forces between the polymer and head-group regions of the SDS clusters. The detailed nature of the polymersurfactant interaction depends upon the interplay among these forces, which one might well expect to undergo interesting changes near the LCST transition of polymeric solutions. This will be the subject of future studies of HPC-surfactant interactions. ${ }^{19}$

\section{REFERENCES}

1. F. M. Winnik, M. A. Winnik, and S. Tazuke, J. Phys. Chem., 91, 594 (1987).

2. For a review on the interactions between surfactants and neutral polymers, see: E. D. Goddard, Colloids and Surfaces, 19, 255 (1986).

3. A. Carlsson, G. Karlström, and B. Lindman, J. Phys. Chem., 93, 3673 (1987).

4. B. Cabane and R. Duplessix, J. Physique, 43, 1529 (1982); ibid., 48, 651 (1987).

5. R. Zana, P. Lianos, and J. Lang, J. Phys. Chem., 89, 41 (1985).

6. F. M. Witte and J. B. F. N. Engberts, Colloids and Surfaces, 36, 417 (1989).

7. N. J. Chang and E. W. Kaler, J. Phys. Chem., 89, 2996 (1985).

8. From: "Atlas of Spectral Data and Physical Constants for Organic Compounds," J. G. Grasselli, Ed., Chemical Rubber Co. Press, Cleveland, Ohio, 1973.

9. For a review, see K. Kalyanasundaram, "Photochemistry in Microheterogeneous Systems," Academic Press, Orlando, Florida, 1987, Chapter 2.

10. R. Zana, in "Surfactant Solutions, New Methods of Investigation," R. Zana, Ed., Marcel Dekker, New York, N.Y., 1985, Chapter 5.

11. F. Grieser and C. J. Drummond, J. Phys. Chem., 92 , 5580 (1988).

12. G. G. Warr and F. Grieser, J. Chem. Soc., Faraday Trans. 1, 82, 1813 (1986).

13. Recently two Swedish groups have also used the benzophenone-pyrene pair to determine $\bar{N}$ and to study fluorescence quenching in micelles: G. Wikander and L. B. Å. Johansson, Langmuir, 5, 728 (1988): M. Almgren, J. Alsins, E. Muhktar, and J. van Stam, in "Reactions in Compartmentalized Liquids," W. Knoche and R. Schomäcker, Ed., 
Springer-Verlag, Berlin, 1989.

14. N. J. Turro and A. Yekta, J. Am. Chem. Soc., 100, 5951 (1978).

15. E. Roelants, E. Gelade, C. Y. Van der Auweraer, and F. C. DeSchryver, J. Colloid Interface Sci., 96, 288 (1983).

16. G. A. F. Roberts and I. M. Thomas, Polymer, 19, 459 (1978).

17. M. G. Wirick and M. H. Waldman, J. Appl. Polym.
Sci., 14, 579 (1970).

18. B. Nystrom and R. Bergman, Eur. Polym. J., 14, 431 (1978).

19. This project was begun in Japan in the laboratories of Professor Shigeo Tazuke, with whom the authors spent a sabbatical leave in $1985 / 86$. We dedicate this paper to Professor Tazuke. With his untimely passing away, we have lost a dear friend and a respected colleague. 